\title{
Some oribatid mites (Acari, Oribatida) from the State of São Paulo, Brazil
}

\author{
Anibal Ramadan Oliveira ${ }^{1}$ \\ Dania Prieto ${ }^{2}$ \\ Gilberto José de Moraes ${ }^{3}$
}

\begin{abstract}
A survey was conducted in three sites from northern and mid-eastern regions of the State of São Paulo, Brazil, to determine oribatid mite composition in patches of seasonal semideciduous forest and soybean crop plantation. A list of 52 taxa, 24 at the species level and 28 only at the genus level, is given, 27 of which are new records for the region.

KEY WORDS. oribatid mites, São Paulo, Brazil, semideciduous forest, soybean
\end{abstract}

Oribatid mites have received considerable attention around the world for their assumed participation in the decomposition process of organic matter and their potential as bioindicators of soil conditions. The knowledge of the taxa found in a given region is important to provide a background for further ecological research.

Most records of oribatid mites in the State of São Paulo, southeast Brazil, derive from samples taken near the city of São Paulo and from the northern coast of the State (SCHUSTER 1962, 1977; MÄrKEL 1964; BECK 1965; GRANDJEAN 1966, 1968; BALOGH \& MAHUNKA 1977, 1978; NiedBala 1981; BALOGH \& BALOGH 1985; PÉREZ-IÑIGO \& BAGGIO 1980, 1988, 1989, 1991, 1993, 1994). Little is known for other regions (SCHUSTER 1962; BECK 1965; BALOGH \& MAHUNKA 1978; KRISPER 1984; PÉrEZ-IÑIGO \& BAGgio 1985, 1986, 1993, 1994; BALOGH 1986; PASCHOAL 1987a,b). There are no records for the northern central part of the State. This paper reports on a survey for oribatid mites at tree sites:

I) a native seasonal semideciduous forest located at Bosque Municipal, São José do Rio Preto, northern State of São Paulo (at 20 48 '36"S and 49²2'50'W). The tree canopy consisted of Copaifera langsdorffii Desf. (Fabaceae), Ficus guaranitica Chod. (Moraceae), Coccoloba latifolia Lam. (Polygonaceae), Myroxylon peruiferum Linn.f. (Fabaceae) and Mabea fistulifera Mart. (Euphorbiaceae), with an under growth composed mainly of Siparuna guianensis Aubl. (Monimiaceae) and

1) Curso de Pós-Graduação em Ciências Biológicas, Área de Zoologia, Instituto de Biociências, Universidade de São Paulo. Caixa Postal 11461, 05422-970 São Paulo, São Paulo, Brazil. E-mail: arolivei@carpa.ciagri.usp.br

2) Departamento de Biologia Animal y Humana, Facultad de Biologia, Universidad de la Habana. Calle 25 No. 555, entre J e I, Vedado, Ciudad de La Habana, Cuba, 10400.

E-mail: dania@fbio.oc.uh.cu

3) Departamento de Entomologia, Fitopatologia e Zoologia Agrícola, Escola Superior de Agricultura "Luiz de Queiroz", Universidade de São Paulo. Caixa Postal 9, 13418-900 Piracicaba, São Paulo, Brazil. E-mail: gjmoraes@carpa.ciagri.usp.br 
Alibertia edulis A.Rich. (Rubiaceae). Litter samples were collected on 10 occasions at approximately two week intervals between late November 1998 and late April 1999;

II) a reclaimed seasonal semideciduous forest formed in an old Eucalyptus spp. (Myrtaceae) plantation located at Jaguariúna, mid-eastern State of São Paulo (at $22^{\circ} 42^{\prime} 18^{\prime \prime} \mathrm{S}$ and $\left.46^{\circ} 59^{\prime} 22^{\prime \prime} \mathrm{W}\right)$. Litter samples were taken in late May 1993 ;

III) a soybean field located at Jaguariúna, in an area which had not been cultivated in the three years period preceeding the samplings. The predominant plant species before soybean plantation were Sida cordifolia L. (Malvaceae), Brachiaria plantaginea Hitchc. (Poaceae) and Panicum maximum Jacq. (Poaceae). Soil samples were taken on five occasions at two week intervals between late January and early April 1997.

Mite extraction was done with Berlese-Tullgren funnels. The specimens were identified using mainly the keys of BALOGH (1972) and BALOGH \& BALOGH $(1988,1990)$. The classification system adopted in this paper is that of FUJIKAWA (1991). New records of genera and species for the State of São Paulo are indicated.

Voucher Specimens are deposited in the Zoology Reference Collection of Escola Superior de Agricultura "Luiz de Queiroz", Universidade de São Paulo, Piracicaba, São Paulo, Brazil.

\section{LIST OF MITES COLLECTED}

Remark. New records for the State of São Paulo are indicated by asterisks. Collection sites are indicated in parentheses after species names by symbols described previously in the text.

Palaeacaridae Grandjean, 1932 [= Ctenacaridae Grandjean, 1954]

Beklemishevia Zachvatkin, 1945 *

B. barbata (Schubart, 1968) (I)

Brachychthoniidae Thor, 1934

Brachychthonius Berlese, 1910 * (III)

Cosmochthoniidae Grandjean, 1947

Cosmochthonius Berlese, 1910 * (I)

Phyllochthoniidae Travé, 1967

Phyllochthonius Travé, 1967 * (III)

Lohmanniidae Berlese, 1916

Papillacarus Kunst, 1959 * (III)

Epilohmanniidae Oudemans, 1916

Epilohmannia Berlese, 1916

E. pallida americana J. Balogh \& Mahunka, $1981 *$ (I, III) 
Phthiracaridae Perty, 1841

Phthiracarus Perty, 1841 (II)

Steganacaridae Niedbala, 1986

Atropacarus (Hoplophorella) Berlese, 1923

A. (H.) cucullatus (Ewing, 1909) * (I, II)

A. (H.) lanceoseta (Balogh \& Mahunka, 1981) * (I)

A. (H.) scapellata (Aoki, 1965) * (I, II, III)

Hoplophthiracarus Jacot, 1933 (II)

Euphthiracaridae Jacot, 1930

Rhysotritia Märkel \& Meyer, 1959 (III)

R. comteae Mahunka, 1983 * (I, II)

Oribotritiidae Grandjean, 1954

Indotritia Jacot, $1928 *$

I. bellingeri Niedbala \& Schatz, 1996 (I)

Allonothridae Lee, 1985

Allonothrus Hammen, 1953

A. neotropicus Balogh \& Mahunka, 1969 * (I)

Nothridae Berlese, 1896

Nothrus C.L. Koch, 1836

N. biciliatus C.L. Koch, 1841 (I, III)

N. becki Balogh \& Mahunka, 1981 * (I)

Pheroliodidae Paschoal, 1987

Pheroliodes Grandjean, 1931 (I, II)

Plateremaeidae Trägardh, 1931

Plateremaeus Berlese, 1908

P. ornatissimus (Berlese, 1888) (II)

Microtegeidae J. Balogh, 1972

Microtegeus Berlese, 1916 (II)

Eremaeozetidae Piffl, 1972

Eremaeozetes Berlese, 1913 * (I)

Microzetidae Grandjean, 1936

Berlesezetes Mahunka, 1980 *

B. brazilozetoides J. Balogh \& Mahunka, 1981 (I, III)

Schizozetes J. Balogh, 1962 * (III)

Damaeolidae Grandjean, 1965

Fosseremus Grandjean, 1954 *

F. saltaensis (Hammer, 1958) (III) 
Eremobelbidae J. Balogh, 1961

Eremobelba Berlese, 1908 (II)

Eremulidae Grandjean, 1965

Eremulus Berlese, 1908 (II, III)

E. rigidisetosus J. Balogh \& Mahunka, 1969 * (I)

Heterobelbidae J. Balogh, 1961

Heterobelba Berlese, 1913 (I)

Astegistidae J. Balogh, 1961

Cultroribula Berlese, 1908 * (II)

Carabodidae C.L. Koch, 1842

Carabodes C.L. Koch, $1836^{*}$

C. atrichosus Mahunka, 1984 (I)

Otocepheidae J. Balogh, 1961

Pseudotocepheus J. Balogh, 1960 (II)

Tectocepheidae Grandjean, 1954

Tectocepheus Berlese, 1896 (I, II, III)

Eremellidae J. Balogh, 1961

Eremella Berlese, 1913 * (I)

Oppiidae Grandjean, 1951

Amerioppia Hammer, 1961 * (I, II)

Brachioppia Hammer, 1961 (I, II)

Mystroppia J. Balogh, 1959 * (III)

Oppiella Jacot, 1937

O. nova (Oudemans, 1902) (I, III)

Ramusella Hammer, 1962 (I, II, III)

Striatoppia J. Balogh, 1958 * (III)

Teratoppia J. Balogh, 1959 (II)

Suctobelbidae Jacot, 1938

Suctobelbella Jacot, 1937 * (II)

S. complexa (Hammer, 1958) (I, III)

S. variosetosa (Hammer, 1961) (I)

Haplozetidae Grandjean, 1936

Haplozetes Willmann, 1935 (I)

Rostrozetes Sellnick, 1925

R. foveolatus Sellnick, 1925 (I, II, III) 
Nasobatidae J. Balogh, 1972

Nasobates Woolley, 1966 * (I)

Oripodidae Jacot, 1925

Oripoda Banks, 1904 (I, II)

Scheloribatidae Grandjean, 1933

Scheloribates Berlese, 1908 (II)

S. praeincisus Berlese, 1910 (I, III)

Xylobatidae J. Balogh \& P. Balogh, 1984

Brasilobates Pérez-Iñigo \& Baggio, 1980

B. bipilis Pérez-Iñigo \& Baggio, 1980 (II)

Xylobates Jacot, 1929 (I)

Ceratozetidae Jacot, 1925

Ceratobates J. Balogh \& Mahunka, 1969 (II)

Oribatellidae Jacot, 1925

Lamellobates Hammer, 1958 (I)

L. molecula (Berlese, 1916) (III)

Galumnidae Jacot, 1925

Erogalumna Grandjean, 1964 * (II)

Galumna Heyden, 1826 (II)

G. glabra Pérez-Iñigo \& Baggio, 1991 (I, III)

Pergalumna Grandjean, 1936 (II)

P. decoratissima Pérez-Iñigo \& Baggio, 1986 (I)

ACKNOWLEDGEMENTS. To Wojciech Niedbala (Department of Taxonomy \& Ecology, A. Mickiewicz University, Poland), for the identification of Steganacaridae and Euphthiracaridae species.

\section{REFERENCES}

BALOGH, J. 1972. The oribatid genera of the world. Budapest, Akadémiai Kiadó, 188p.

Balogh, J. \& P. Balogh. 1985. Fifteen new species of the genus Xenillus Robineau-Desvoidy, 1839

(Acari: Oribatei) from South America. Acta Zool. Hung. 31 (1-3): 53-79.

- 1988. The soil mites of the world. 2. Oribatid mites of the Neotropical Region I. Amsterdam, Elsevier, 335p.

- 1990. The soil mites of the world. 3. Oribatid mites of the Neotropical Region II. Amsterdam, Elsevier, 333p.

BALOGH, J. \& S. MAHUNKA. 1977. New data to the knowledge of the oribatid fauna of Neogea (Acari). I. Acta Zool. Hung. 23 (1-2): 1-28.

1978. New data to the knowledge of the oribatid fauna of Neogea (Acari). III. Acta Zool. Hung. 24 (3-4): 269-299.

BALOGH, J. 1986. Four new Xenillus species from the Neotropical Region (Acari: Oribatei).

Opusc. Zool. Budapest. 22: 43-50. 
BECK, L. 1965. Über Variabilität und Wertigkeit morphologischer Merkmale bei adulten Oribatiden (Arachnida, Acari): am Beispel der Gattung Rostrozetes Sellnick, 1925. Abh. senckenb. naturf. Ges. 508: 1-64.

FUJIKAWA, T. 1991. List of oribatid families and genera of the world. Edaphologia 46: 1-130.

Grandjean, F. 1966. Les Staurobatidae n. fam. (Oribates). Acarologia 8 (4): 696-727.

- 1968 Schusteria littorea n. g., n. sp. et les Selenoribatidae (Oribates). Acarologia 10 (1): 116-150.

KRISPER, G. 1984. Zetorchestes schusteri n. sp. - Erstnachweis der Milbenfamilie Zetorchestidae in Südamerika (Acari, Oribatei). Mitt. Hamburg. Zool. Mus. u. Inst. 81: 177-187.

MÄrKEL, K. 1964 Die Euphthiracaridae Jacot, 1930, und ihre Gattungen (Acari, Oribatei). Zool. Verhand. 67: 1-78.

Niedbala, W. 1981. Trois nouveaux Phthiracaridae (Acari, Oribatida) originaires du Brésil. Acarologia 23 (1): 63-80.

PASCHOAL, A.D. 1987a. A revision of the Plateremaeidae (Acari: Oribatei). Revta bras. Zool. 3 (6): 327-356. 357-384

Pérez-IÑigo, C. \& D. Baggio. 1980. Oribátidos edáficos do Brasil. I. Bol. Zool. Univ. São Paulo 5: 111-147.

. 1985. Oribates édaphiques du Brésil (II). Oribates de l'Île du "Cardoso" (Première Partie). Acarologia 26 (2): 183-199.

- 1986. Oribates édaphiques du Brésil (III). Oribates de l'Île du "Cardoso" (Deuxième Partie). Acarologia 27 (2): 163-179.

— 1988. Oribates édaphiques du Brésil (IV). Oribates de l'état de São Paulo (Première Partie). Acarologia 29 (2): 189-204.

— 1989. Oribates édaphiques du Brésil (V). Oribates de l'état de São Paulo (Deuxième Partie). Acarologia 30 (3): 261-274. . 1991. Oribates édaphiques du Brésil (VI). Oribates de l'état de São Paulo (Troisième Partie). Acarologia 32 (1), 79-92.

- 1993. Oribates édaphiques du Brésil (VII). Oribates de l'état de Sao Paulo (Quatrieme Partie). Acarologia 34 (3): 249-264.

- 1994. Oribates édaphiques du Brésil (VIII). Oribates de l'état de São Paulo (Cinquieme Partie). Acarologia 35 (2): 181-198.

Schuster, R. 1962. Neue Mesoplophora-vorkommen in der Neotropis (Arach., Acari, Oribatei).

Senckenb. Biol. 43 (6): 489-495. 1977. Die Selenoribatidae, eine thalassobionte Familie der Hornmilben (Oribatei). Acarologia 19 (1): 155-160.

Recebido em 24.VIII.2000; aceito em 26.VI. 2001. 